\title{
Influencia del apoyo del acompañante durante el trabajo de parto en un Hospital de Huancayo
}

\author{
Influence of the companion's support during labor in a hospital in Huancayo \\ Mirian Carrillo Cayllahua ${ }^{1, a}$, Melva Medaline Vera de la Cruz ${ }^{1, b}$, Guido F. Marín ${ }^{2, c}$ \\ RESUMEN
}

Objetivo: Determinar la influencia del apoyo del acompañante durante el trabajo de parto en el Hospital Regional Docente Materno Infantil El Carmen. Material y Métodos: Estudio de tipo sustantiva, retrospectiva, de nivel descriptivo correlacional de corte transversal prospectivo, tuvo una población de 362 gestantes en trabajo de parto, del cual se obtuvo la muestra mediante criterios de inclusión y exclusión, obteniendo la muestra de 98 gestantes en trabajo de parto, para la medición de las variables se empleó la técnica de la observación y análisis documental. Resultados: El $42,9 \%$ son primigestas y $57,1 \%$ son multigestas, así mismo, del total de partos atendidos se generaron $61,2 \%$ con acompañante y $38,8 \%$ sin acompañante; de los partos con acompañante se tuvo como parentesco de mayor frecuencia a la pareja con $52.0 \%$, seguido de la madre con $6,1 \%$, otros (padre, hermano, amigos) 2,0\% y por último la hermana con 1,0\%. Conclusiones: El acompañamiento familiar durante el trabajo de parto favorece a los beneficios maternos en todas sus dimensiones; en relación a los beneficios perinatales solo favorece en la frecuencia cardíaca fetal y no genera beneficio a las demás dimensiones estudiadas con un nivel de significancia al 0,05 .

PALABRAS CLAVE: Acompañante, influencia, trabajo de parto.

\section{SUMMARY}

Objective: To determine the influence of the companion's support during labor at the Hospital Regional Docente Materno Infantil El Carmen. Material and Methods: Substantive, retrospective, descriptive, correlational, prospective cross-sectional study, with a population of 362 pregnant women in labor, from which the sample was obtained through inclusion and exclusion criteria, obtaining a sample of 98 pregnant women in labor, for the measurement of the variables the technique of observation and documentary analysis was used. Results: $42.9 \%$ were primigravida and $57.1 \%$ were multigestational, and of the total number of deliveries attended, $61.2 \%$ were with an attendant and $38.8 \%$ without; of the deliveries with an attendant, the most frequent relationship was the couple with $52.0 \%$, followed by the mother with $6.1 \%$, others (father, brother, friends) with $2.0 \%$ and finally the sister with $1.0 \%$. Conclusions: Family accompaniment during labor favors maternal benefits in all its dimensions; in relation to perinatal benefits, it only favors fetal heart rate and does not benefit the other dimensions studied with a significance level of 0.05 .

KEYWORDS: Accompanying person, influence, labor.

\footnotetext{
Universidad Privada de Huancayo Franklin Roosevelt. Huancayo, Perú.

Universidad Nacional de Huancavelica. Huancavelica, Perú.

Obstetra, Magister en Ciencias de la Salud Mención Salud Pública. ORCID ID: 0000-0003-4150-9877

Obstetra, Magister en Gestión en Servicios de la Salud. ORCID ID: 0000-0002-5174-5622

Lic. Enfermería, Magister en Ciencias de la Salud Mención Salud Pública ORCID ID: 0000-0003-0213-8225
} 


\section{INTRODUCCIÓN}

El presente artículo se refiere al tema de apoyo del acompañante durante el trabajo de parto, que se define como el "proceso por el cual la gestante recibe asistencia, cuidado y compañía de una persona de su elección durante el trabajo de parto, parto y puerperio" (1). El embarazo y en especial el parto representan un momento crítico en la mujer desde una vista psicológica, ya que puede resultar ser un acontecimiento estresante debido al nivel alto de ansiedad, tensión, desconfianza y miedo que este proceso puede generar en la mujer $(2,3)$, la cual hace que sea más vulnerable influyendo negativamente en el carácter y duración del parto y aumentando la posibilidad de presentar complicaciones obstétricas. Es por ello, que durante el proceso del trabajo de parto se debe asegurar que el ambiente ofrezca confianza, privacidad, comunique respeto y eviten factores estresantes; del mismo modo es necesario que la mujer cuente con el apoyo de un acompañante; pudiendo ser éste, la pareja, la madre, la hermana, o cualquier otro familiar o amigo de elección $(4,5)$. El apoyo del acompañante durante el trabajo de parto podría resultar beneficioso durante el desarrollo del mismo, al encontrarse en un entorno agradable, de apoyo íntimo (6). Por lo anteriormente mencionado y teniendo en cuenta que las mujeres deben elegir a la persona que les proporcionara apoyo durante el trabajo de parto y teniendo en cuenta que en la actualidad existe poca información e investigaciones que nos permitan identificar cuál es la influencia del apoyo del acompañante durante el trabajo de parto y cómo es dicho apoyo amerita plantear nuevos estudios.

Dentro de la realidad problemática mencionaremos que desde el año 1985, y hasta la fecha, la OMS recomienda intervenir en el parto solo de ser necesario, debido a que el parto normal es un proceso saludable (7). Así mismo la OMS publicó la Declaración de Fortaleza; documento en el cual se recomienda “... Estimular la presencia de una persona que la mujer elija para que la acompañe durante el trabajo de parto y el parto" (8).

Así mismo, Mucio et al., (9), recomiendan la presencia de un acompañante durante el trabajo de parto debido a que mejora los resultados maternos y perinatales y la satisfacción de las mujeres con los servicios de salud. Además, uno de los factores que generan el no cumplimiento de consignar un acompañante en el trabajo de parto es que los profesionales de la partería no siempre conocían la política o el protocolo de su país para implementarla (10).
En España recientemente, se dio a conocer que las medidas llevadas a cabo en un Hospital de Zaragoza se están optando por la separación de la mujer de su acompañante durante la fase de dilatación debido al contexto de la pandemia actual. Sin embargo; esta medida, niega el derecho de la mujer a permanecer acompañada durante la dilatación evitando los beneficios que afirma la OMS de contar con la pareja en este proceso (11).

En muchos países de América Latina, el acompañamiento durante el parto está legalmente asegurada por normas, actualmente vigentes, que promueven el parto humanizado (12); tales son los casos de Puerto Rico (Ley Núm. 156 del año 2006), Uruguay (Ley N ${ }^{\circ} 17.386$ del año 2001), y Argentina que cuenta con la Ley $\mathrm{N}^{\circ} 25.929$ desde el año 2004, donde menciona que toda mujer "tiene derecho a estar acompañada por una persona de su confianza y elección en el momento del parto" $(13,14)$.

En el año 2015, el estado peruano aprobó el proyecto de Ley 1158/2011-CR, que consiste en la promoción y protección del derecho a un parto humanizado y a la salud de la mujer gestante y el recién nacido, haciendo mención en el artículo 3 : "Toda mujer durante el desarrollo del embarazo, trabajo de parto, parto y postparto, tiene los siguientes derechos: ...A ser acompañada por una persona de su elección y confianza durante el trabajo de parto, parto y post parto, garantizándose este derecho a través de las recomendaciones y cumplimiento de los protocolos a que hubiera lugar" (15).

Ley que se viene aplicando en algunas instituciones prestadoras de servicios de salud incluso mucho antes de su vigencia, ya sea como parte cultural del proceso reproductivo, o algo más estructurado como el caso del Instituto Nacional Materno Perinatal (INMP) de Lima, que tiene implementado los programas Parto con acompañante y Papá canguro desde el año 2007 gracias al cual durante el año 2015 se produjeron más de ocho mil partos con la presencia de papás (15). “... El número de partos con acompañante en el INMP se ha incrementado en más de 10 veces desde su implementación, esto representa para el 2016 el 49\% de un total de 11047 partos anuales..." (16).

A pesar de ello, existen reducidos estudios que revelen cuál es el apoyo brindado por el acompañante (pareja, madre, suegra, u otro familiar) y cómo influye éste en el trabajo de parto; sea beneficiando o no al binomio madre-hijo (17). Sin embargo, se ha 
revisado algunos artículos en el que se considera que el trabajo de parto con acompañante puede resultar muy beneficioso para una mujer, al encontrarse en un entorno agradable, de apoyo íntimo. A pesar de la vigencia de la Ley del parto humanizado, aún se observa un porcentaje de mujeres que no cuentan con el apoyo de un acompañante durante su trabajo de parto.

\section{MATERIAL Y MÉTODOS}

Investigación de tipo Sustantiva, prospectivo (18), de nivel descriptivo, porque se buscó describir la influencia del apoyo del acompañante durante el trabajo de parto, se tuvo una población de 362 gestantes en trabajo de parto atendidas en el Hospital Regional Docente Materno Infantil El Carmen durante los meses de Julio a Setiembre de 2021, se obtuvo la muestra mediante criterios de inclusión y exclusión con un muestreo por criterio siendo 98 gestantes incluidas en el estudio.

Los instrumento de recolección de datos fueron la ficha de observación y análisis documental (19), para apoyo del acompañante (5 ítems) la cual fue validada mediante juicio de expertos y su estadística $\mathrm{V}$ de Aiken, obteniendo el valor de 0.88 , lo que indica una adecuada validez y para la confiabilidad se empleó el Alpha de Cronbach, obteniendo un valor de 0.90 que indica fiabilidad de contenido aceptable de los ítems propuestos; para la variable influencia maternoperinatal (12 ítems) no se requirió validación ni confiabilidad por ser el instrumento la ficha de análisis documental.

Tabla 1. Tipo de gestación de pacientes en trabajo de parto atendidos en el Hospital Regional Docente Materno Infantil El Carmen atendidas.

\begin{tabular}{ccc}
\hline Número de gestas & f & $\mathbf{\%}$ \\
\hline Primigesta & 42 & 42,9 \\
Multigesta & 56 & 57,1 \\
Total & 98 & 100,0 \\
\hline
\end{tabular}

Fuente: Elaboración propia, producto del instrumento aplicado.

Tabla 2. Trabajos de parto con acompañantes atendidos en el Hospital Regional Docente Materno Infantil El Carmen.

\begin{tabular}{ccc}
\hline Partos & F & $\mathbf{\%}$ \\
\hline Sin Acompañante & 38 & 38,8 \\
Con Acompañante & 60 & 61,2 \\
Total & 98 & 100,0 \\
\hline Fuente: Elaboración propia, producto del instrumento aplicado.
\end{tabular}

La aplicación de los instrumentos se realizaron in situ previa autorización por las autoridades correspondientes del Hospital Regional Docente Materno Infantil El Carmen; así mismo se aplicó el consentimiento informado a los acompañantes de las gestantes en trabajo de parto.

Para el análisis estadístico se empleó el programa SPSS v.26 para Windows 10. Empleando la estadística descriptiva para el análisis de datos y para la contratación de la hipótesis se empleó la estadística inferencial no paramétrica, debido a que la prueba de normalidad no aplica para variables cualitativas; siendo el estadígrafo aplicado el Chi Cuadrado (20).

\section{RESULTADOS}

Del total de gestantes en trabajo de parto atendidas en el Hospital Regional Docente Materno Infantil "El Carmen", 42,9\% son primigestas y $57 ., 1 \%$ son multigestas (tabla 1).

Del total de partos atendidos se generaron $61,2 \%$ con acompañante y $38,8 \%$ sin acompañante (tabla 2 ).

De los partos con acompañante se tuvo como parentesco de mayor frecuencia a la pareja con 52,0\%, seguido de la madre con $6,1 \%$, otros (padre, hermano, amigos) $2.0 \%$ y por último la hermana con $1.0 \%$ (tabla $3)$.

Respecto a los beneficios maternos que genera el acompañante durante los procesos de parto se establece que existe menor frecuencia de episiotomía, desgarro perineal, complicaciones en el periodo expulsivo, complicaciones en el periodo de alumbramiento con un valor de significancia de $0.000 ; 0,016 ; 0,01 ; 0,012$ respectivamente; menor tiempo de dilatación con un valor de significancia de 0,001 y menor frecuencia

Tabla 3. Parentesco del acompañante de los trabajos de parto atendidos en el Hospital Regional Docente Materno Infantil El Carmen.

\begin{tabular}{ccc}
\hline Parentesco & F & \% \\
\hline Pareja & 51 & 52,0 \\
Madre & 6 & 6,1 \\
Hermana & 1 & 1,0 \\
Otros & 2 & 2,0 \\
Sin Acompañante & 38 & 38,8 \\
Total & 98 & 100,0 \\
\hline Fuente: Elaboración propia, producto del instrumento aplicado.
\end{tabular}


Tabla 4. Beneficios maternos del acompañante durante el trabajo de parto en el Hospital Regional Docente Materno Infantil El Carmen

\begin{tabular}{|c|c|c|c|c|c|c|c|c|}
\hline \multirow{2}{*}{$\mathbf{F}$} & & \multicolumn{2}{|c|}{ Sin Acompañante } & \multicolumn{2}{|c|}{ Con Acompañante } & \multirow{2}{*}{ Total } & \multirow{2}{*}{$\%$} & \multirow{2}{*}{$\begin{array}{c}\text { Nivel de } \\
\text { Significancia }\end{array}$} \\
\hline & & $\%$ & $\mathbf{F}$ & $\%$ & & & & \\
\hline \multirow{2}{*}{ Episiotomía } & $\mathrm{Si}$ & 14 & 14,9 & 4 & 4,08 & 18 & 18,37 & \multirow{2}{*}{0,000} \\
\hline & No & 24 & 24,49 & 56 & 57,14 & 80 & 81,63 & \\
\hline \multirow{2}{*}{ Desgarro perineal } & $\mathrm{Si}$ & 9 & 9,18 & 4 & 4,08 & 13 & 13,27 & \multirow{2}{*}{0,016} \\
\hline & No & 29 & 29,59 & 56 & 57,14 & 85 & 86,73 & \\
\hline \multirow{2}{*}{ Dilatación } & $\mathrm{Si}$ & 10 & 10,20 & 2 & 2,04 & 12 & 12,24 & \multirow{2}{*}{0,001} \\
\hline & No & 28 & 28,57 & 58 & 59,18 & 86 & 87,76 & \\
\hline \multirow{2}{*}{$\begin{array}{l}\text { Complicaciones en el } \\
\text { Periodo Expulsivo }\end{array}$} & $\mathrm{Si}$ & 4 & 4,08 & 0 & 0,00 & 4 & 4,08 & \multirow{2}{*}{0,01} \\
\hline & No & 34 & 34,69 & 60 & 61,22 & 94 & 95,92 & \\
\hline \multirow{2}{*}{$\begin{array}{l}\text { Complicaciones en el } \\
\text { Periodo de Alumbramiento }\end{array}$} & $\mathrm{Si}$ & 7 & 7,14 & 2 & 2,04 & 9 & 9,18 & \multirow{2}{*}{0,012} \\
\hline & No & 31 & 31,63 & 58 & 59,18 & 89 & 90,82 & \\
\hline \multirow{2}{*}{ Terminación del parto } & Vaginal & 34 & 34,69 & 60 & 61,22 & 94 & 95,92 & \multirow{2}{*}{0,01} \\
\hline & Cesare & 4 & 4,08 & 0 & 0,00 & 4 & 4,08 & \\
\hline Total & & 38 & 38,78 & 60 & 61,22 & 98 & 100,00 & \\
\hline
\end{tabular}

Fuente: Elaboración propia, producto del instrumento aplicado.

Tabla 5. Beneficios perinatales del acompañante durante el trabajo de parto en el Hospital Regional Docente Materno Infantil El Carmen.

\begin{tabular}{|c|c|c|c|c|c|c|c|c|}
\hline \multirow{2}{*}{ f } & & \multicolumn{2}{|c|}{$\begin{array}{c}\text { Sin } \\
\text { Acompañamiento }\end{array}$} & \multicolumn{2}{|c|}{$\begin{array}{c}\text { Con } \\
\text { Acompañamiento }\end{array}$} & \multirow[t]{2}{*}{ Total } & \multirow[t]{2}{*}{ f } & \multirow{2}{*}{$\begin{array}{c}\text { Nivel de } \\
\text { Significancia }\end{array}$} \\
\hline & & $\%$ & f & f & $\%$ & & & \\
\hline \multirow{2}{*}{$\begin{array}{l}\text { Frecuencia Cardiaca } \\
\text { Fetal }\end{array}$} & Taquicardia & 7 & 7,14 & 0 & 0,00 & 7 & 7,14 & \multirow{2}{*}{0,001} \\
\hline & Normal & 31 & 31,63 & 60 & 61,22 & 91 & 92,86 & \\
\hline \multirow{2}{*}{ Apgar al minuto } & 4 a 6 & 2 & 2,04 & 0 & 0,00 & 2 & 2,04 & \multirow{2}{*}{0,073} \\
\hline & 7 a 10 & 36 & 36,73 & 60 & 61,22 & 96 & 97,96 & \\
\hline \multirow{2}{*}{ Apgar a los 5 minutos } & 4 a 6 & 2 & 2,04 & 0 & 0,00 & 2 & 2,04 & \multirow{2}{*}{0,073} \\
\hline & 7 a 10 & 36 & 36,73 & 60 & 61,22 & 96 & 97,96 & \\
\hline \multirow{2}{*}{ RCP Neonatal } & $\mathrm{Si}$ & 2 & 2,04 & 0 & 0,00 & 2 & 2,04 & \multirow{2}{*}{0,073} \\
\hline & No & 36 & 36,73 & 60 & 61,22 & 96 & 97,96 & \\
\hline \multirow{2}{*}{ Trauma Obstétrico } & $\mathrm{Si}$ & 4 & 4,08 & 1 & 1,02 & 5 & 5,10 & \multirow{2}{*}{0,052} \\
\hline & No & 34 & 34,69 & 59 & 60,20 & 93 & 94,90 & \\
\hline \multirow{2}{*}{$\begin{array}{l}\text { Complicaciones } \\
\text { Neonatales tempranas }\end{array}$} & $\mathrm{Si}$ & 2 & 2,04 & 0 & 0,00 & 2 & 2,04 & \multirow{2}{*}{0,073} \\
\hline & No & 36 & 36,73 & 60 & 61,22 & 96 & 97,96 & \\
\hline Total & & & & & & 98 & 100,00 & \\
\hline
\end{tabular}

Fuente: Elaboración propia, producto del instrumento aplicado.

de terminación del parto vía cesárea con un valor de significancia de 0,01 (tabla 4).

Con respecto a beneficios perinatales del acompañante durante el parto se evidencia que favorece a una frecuencia cardiaca fetal normal con un valor de significancia de 0,001 ; no beneficiando en el Apgar al minuto, a los 5 minutos, en la presencia de RCP neonatal, trauma obstétrico y complicaciones neonatales tempranas, con un valor de significancia 0,$073 ; 0,073 ; 0,073 ; 0,052 ; 0,073$ respectivamente (tabla 5). 


\section{DISCUSIÓN}

Los resultados muestran, las gestantes que ingresaron al Hospital Regional Docente Materno Infantil "El Carmen" para el proceso de trabajo de parto, fueron más multigestas, que primigestas, existe mayor cantidad de gestantes en trabajo de parto en quienes ingresaron con acompañante $\mathrm{y}$ menor frecuencia de gestantes en trabajo de parto en quienes acudieron sin acompañante. Estos datos son alarmantes y toman importancia ante lo mencionado por organismos internacionales como la OMS (7), que a pesar de recomendar el acompañamiento no se cumpla a cabalidad, existiendo aun gestantes en trabajo de parto sin acompañantes, estos hechos pueden deberse a lo manifestado por el Ministerio de Sanidad y Política Social (10), que expresa que no se cumple lo establecido en la norma legal por desconocimiento del profesional que se dedica a la partería evitando la presencia del acompañante. De gestantes en trabajo de parto que ingresaron con acompañante la mayoría es su pareja, seguida de su madre, hermana y en menor proporción otros (padre, hermano, amigos). Todos estos datos toman relevancia con lo expresado por la Organización Mundial de la Salud que manifiesta dentro de sus recomendaciones el acompañamiento durante el trabajo de parto y el parto por la persona de su elección siendo (pareja, padres, familia o persona elegida por la gestante) (7). Así mismo, es reforzado por Matute (8), quién manifiesta que el profesional de la salud debe de estimular a que la gestante en trabajo de parto elija un acompañante que este a su lado durante dicho acto. En tanto, los resultados que establecen beneficio materno reflejan que sí existe beneficios maternos como menor frecuencia de episiotomía, desgarro perineal, complicaciones en el periodo expulsivo, complicaciones en el periodo de alumbramiento, menor tiempo de dilatación, menor frecuencia de terminación del parto vía cesárea, los datos reflejan lo manifestado por la Organización Mundial de la Salud la cual expresa la existencia de beneficios maternos, también es reforzado por Cantero y Borges et al., $(13,14)$ que expresan que toda mujer tiene derecho a estar acompañada para mejorar su estado psicológico y físico, Hodnett et al., (17) también expresa que existe beneficio al binomio madre-hijo y en relación a beneficios perinatales solo un factor refleja beneficio, siendo que favorece la frecuencia cardiaca fetal normal, en relación a las otras dimensiones no se evidencia beneficio en el Apgar al minuto, a los 5 minutos, en la presencia de RCP neonatal, trauma obstétrico y complicaciones neonatales tempranas. Estos datos se contraponen en forma indirecta a lo mencionado por la OMS, en vista que no específica los beneficios perinatales y las hace de forma genérica. Los autores estamos en concordancia que el acompañamiento durante el trabajo de parto genera beneficios maternos y respecto a los beneficios perinatales pudieran no estar presentes debido a múltiples factores como genéticos, biológicos, e institucionales.

\section{CONCLUSIONES}

El acompañamiento familiar durante el trabajo de parto favorece a los beneficios maternos en todas sus dimensiones; en relación a los beneficios perinatales solo favorece en la frecuencia cardíaca fetal y no genera beneficio a las demás dimensiones perinatales estudiadas con un nivel de significancia al 0,05.

Agradecimiento: A los profesionales de la salud del Hospital Regional Docente Materno Infantil "El Carmen" por el apoyo en el acceso y obtención de datos.

\section{Correspondencia:}

Mirian Carrillo Cayllahua

Correo electrónico: mcarrillo@uroosevelt.edu.pe

\section{REFERENCIAS BIBLIOGRÁFICAS}

1. Ramírez H, Rodríguez I. Beneficios del acompañamiento a la mujer por parte de su pareja durante el embarazo,el parto y el puerperio en relación con el vínculo paternofilial. Matronas. 2014; 15(4).

2. Gonzales J, Del Sol J. Obstetricia. 4th ed. Barcelona: Salvat; 2002.

3. Smith R. Obstetricia, Ginecología y Salud de la Mujer. Barcelona: Masson Editores; 2006.

4. Biurrun A. La humanización de la asistencia al parto: Valoración de la satisfacción, autonomía y del autocontrol. Tesis Doctoral. Barcelona, España: Universitat de Barcelona; 2017.

5. Maldonado-Durán M. Salud Mental Perinatal. Washington DC: Organización Panamericana de la Salud; 2011.

6. Marín D, Bullones Á, Javier F. Influencia de los factores psicológicos en el embarazo, parto y puerperio.Un estudio longitudinal. Nure Investigation. 2008; 37(8).

7. Organización Mundial de la Salud. Recomendaciones de la OMS: Para los cuidados durante el parto, para una experiencia de parto positiva. Washington DC: Organización Mundial de la Salud; 2018.

8. Matute J. Recomendaciones de la OMS para el parto: conflicto de intereses en la práctica. Medicina 
Naturista.2006; 10: 55-59.

9. Mucio B, Binfa L, Ortiz J, Portela A. Situación de la política nacional sobre el acompañante durante el parto en América Latina y el Caribe: brechas y desafíos. Pan American Journal of Public Health. 2020; 44(19). DOI: 10.26633/RPSP.2020.19

10. Ministerio de Sanidad y Política Social. Guía de Práctica Clínica sobre la Atención al Parto Normal. Bizkaia: Servicio Central de Publicaciones del Gobierno Vasco; 2010. (Citado el 15 de junio del 2021). Disponible en: https://portal.guiasalud.es/ wp-content/uploads/2018/12/GPC 472_Parto Normal_Osteba_compl.pdf

11. Asociación española de psicología Perinatal. Sociedad Marcé Española. MARES Acompañamiento a las mujeres en el trabajo de parto: posicionamiento de la AEPP y La Mares. España: Sociedad Marcé Española; 2020. (Citado el 15 de junio del 2021). Disponible en: https:/www.sociedadmarce.org/ detall.cfm/ID/14929/ESP/acompanyamiento-lasmujeres-trabajo-parto-posicionamiento-aepp-ymares-htm

12. Silin J. De mujeres, matronas, doulas y partos. Jaén: Asociación de Amigos del Archivo Histórico Diocesano de Jaén; 2010. (Citado el 15 de junio del 2021). Disponible en: https://dialnet.unirioja.es/ servlet/articulo? codigo $=4095877$.
13. Cantero A, Fiuri A. Acompañamiento en Sala de Partos: Regla o excepción. Revista del Hospital Materno Infantil Ramón Sardá. 2010; 29(3): 108-112.

14. Borges L, Sánchez R, Domínguez R, Sixto A. El parto humanizado como necesidad para la atención integral a la mujer. Rev. Cubana Obstetricia y Ginecología. 2018; 44(3).

15. Congreso de la Republica. Proyecto de Ley $\mathrm{N}^{o}$ 11587211. Lima: Gaceta Juridica; 2019.

16. Ministerio de Salud. Boletín estadístico de Nacimientos del Perú. Lima: Ministerio de Salud; 2016.

17. Bohren MA, Hofmeyr G, Sakala C, Fukuzawa RK, Cuthbert A. Continuous support for women during childbirth. Madrid: La Biblioteca Cochrane Plus; 2016. (Citado el 15 de junio del 2021). Disponible en: http://www.cochrane.org/CD003766/PREG continuous-support-for-women-during-childbirth.

18. Hernandez R, Fernandez C, Baptista P. Metodología de la investigación. Ciudad de Mexico: Mc Graw Hill; 2014.

19. Sánchez CRM. Metodología y diseños en la investigación científica. Lima, Perú: Editorial Mantaro; 2015.

20. Vilalta C. Análisis de Datos. Ciudad de Mexico: Centro de Investigación y Docencia Económicas; 2016.

Recibido: 29/03/2021

Aceptado: 18/10/2021 